\title{
Experimental study of the relationship between fluid density and saturation and sonic wave velocity of rock samples from the WXS Depression, South China Sea
}

\author{
Pei Fagen ${ }^{1}$, Zou Changchun ${ }^{1 *}, \mathrm{He}^{\text {Tao }}{ }^{2}$, Pan Lingzhi ${ }^{1}$, Xiao Kun ${ }^{1}$, Shi Ge ${ }^{2}$ and \\ Ren Keying ${ }^{3}$ \\ ${ }^{1}$ Key Laboratory of Geo-detection (China University of Geosciences, Beijing), Ministry of Education, Beijing 100083 , \\ China \\ ${ }^{2}$ School of Earth and Space Sciences, Peking University, Beijing 100871, China \\ ${ }^{3}$ Zhanjiang Branch of China National Offshore Oil Corporation Ltd., Guangdong 524057, China
}

(c) China University of Petroleum (Beijing) and Springer-Verlag Berlin Heidelberg 2011

\begin{abstract}
The relationship between fluid density and saturation and sonic wave velocity of rock samples taken from the WXS Depression in the South China Sea was studied by an oil-water replacement experiment under simulated in-situ temperature and pressure conditions. Two kinds of low-density oils $\left(0.691\right.$ and $\left.0.749 \mathrm{~g} / \mathrm{cm}^{3}\right)$ and two kinds of high-density oils $\left(0.834\right.$ and $\left.0.873 \mathrm{~g} / \mathrm{cm}^{3}\right)$ were used to saturate the rock samples at different oil-saturation states, and the saturated P- and S-wave velocities were measured. Through Gassmann's equation, the theoretical P- and S-wave velocities were also calculated by the fluid replacement method. With the comparison of the measured values and the theoretical values, this study comes to the following conclusions. (1) With the increase of oil saturation and the decrease of water saturation, the P-wave velocity of rock samples saturated by low-density oil increases and the changing rule is in accord with the effective fluid theory; the P-wave velocity of rock samples saturated by high-density oil decreases and the changing rule goes against the theory. (2) With the increase of oil density (namely $0.691 \rightarrow 0.749 \rightarrow 0.834 \rightarrow 0.873 \mathrm{~g} / \mathrm{cm}^{3}$ ) when oil saturation is unchanged, P-wave velocity increases gradually. (3) The S-wave velocity is always stable and is not affected by the change of oil density and saturation. The results can be used to constrain pre-stack seismic inversion, and the variation rule of sonic wave velocity is valuable for hydrocarbon identification in the study area.
\end{abstract}

Key words: Rock physics, saturation, compressional wave, shear wave, water drive, Gassmann's equation, fluid replacement

\section{Introduction}

Seismic rock physics mainly studies rock and fluid properties and their relationships with geological attribute parameters, and was not developed in China until the end of 1980s. It can effectively guide lithology identification and fluid prediction, oil and gas exploitation, and reservoir monitoring. It is based on sonic wave velocity experiments of rocks, and the influencing factors of sonic wave velocities are complex. In a specific reservoir, the porosity, content of clay and pore fluid saturation of rocks are the key factors which affect sonic wave velocities. In previous research, the rock physics experiments were mostly focused on the changing rule of sonic wave velocities of rocks with different gas/water

*Corresponding author. email: zoucc@cugb.edu.cn

Received March 1, 2010 saturations, and the following conclusions were obtained. (1) In the case of low water saturation, with the increase of water saturation, P-wave velocity of samples changed irregularly, probably due to the uneven distribution of pore fluid and experimental frequency. (2) In the case of high water saturation, with the increase of water saturation, P-wave velocity of samples increased (Domenico, 1974; Knight and Nolen-Hoeksema, 1990; Shi and Shen, 1990; Liu et al, 1994; Zhang and Li, 1995; Goertz and Knight, 1998; Zhao et al, 1998; Jiang and Shi, 1998; Zhang et al, 2000; Shi et al, 2003; Hu et al, 2004; Jiang et al, 2004; Deng et al, 2005). However, the results of rock physics experiments under the condition of different oil/water saturations are seldom reported. Shen and Shi (1994) used a mineral oil with a density of $0.806 \mathrm{~g} / \mathrm{cm}^{3}$ to study the elastic parameters of rocks under different oil/water saturations. Xie et al (1997) made an oil-water displacement experiment using an oil with a density of $0.84 \mathrm{~g} / \mathrm{cm}^{3}$ (a 
mixture of mineral oil and kerosene). Zhao et al (1998) also conducted an experiment on different oil/water saturations. $\mathrm{Xu}$ et al (2000) carried out an experiment using a crude oil with a density of $0.89 \mathrm{~g} / \mathrm{cm}^{3}$. The reported experiments were all carried out with the same oil density. In this study, we use four kinds of oils to analyze the characteristics of P- and S-wave velocities of rocks under different oil/water saturations.

\section{Measurement of sonic wave velocity}

\subsection{Basic information of rock samples}

Four rock samples were selected from more than 40 sandstone cores collected from the WXS Depression in the South China Sea, namely W1, W7, W10 and W11. They were saturated by different kinds of oils and the sonic wave velocities were measured under the following conditions: (1) different oil/water saturations and the same oil density; (2) different oil densities and the same oil/water saturation.

Through the regular physical testing and lithology observation of thin sections under optical microscope, the basic physical parameters of rock samples were obtained, as shown in Table 1.

Table 1 Basic physical parameters of rock samples

\begin{tabular}{cccccccc}
\hline I.D. & Lithology & Depth, $\mathrm{m}$ & Diameter, $\mathrm{mm}$ & Length, $\mathrm{mm}$ & Porosity, $\%$ & Permeability, $\mathrm{mD}$ & $\rho_{\text {dry }}, \mathrm{g} / \mathrm{cm}^{3}$ \\
\hline W1 & Fine sandstone & 2508.6 & 25.72 & 44.95 & 18.45 & 44.62 & 2.10 \\
W7 & Fine sandstone & 2347.17 & 25.72 & 40.3 & 16.46 & 3.87 & 2.13 \\
W10 & Fine sandstone & 2478.35 & 25.64 & 49.82 & 13.55 & 2.49 & 2.24 \\
W11 & Medium sandstone & 2658.33 & 25.70 & 41.21 & 17.87 & 63.55 & 2.05 \\
\hline
\end{tabular}

Note: $\rho_{\text {dry }}$ is the density of dry sample

\subsection{Experimental apparatus}

With the multifunctional acoustic parameter automatic measurement system AUTOLAB-1000, the P- and S-wave velocities of rock samples were measured at simulated insitu temperature and pressure. A maximum temperature of $125^{\circ} \mathrm{C}$ is provided by the device and the deviation of the Omega temperature sensor is below $0.1{ }^{\circ} \mathrm{C}$; the maximum confining pressure is $103.4 \mathrm{MPa}$, the maximum pore pressure is $68.95 \mathrm{MPa}$, and the accuracy of pressure sensor is $0.25 \%$. The ultrasonic pulse transmission method is used to measure $\mathrm{P}$ - and S-wave velocities in the experiment. The center frequency of $\mathrm{P}$-wave and $\mathrm{S}$-wave is $700 \mathrm{kHz}$ and $200 \mathrm{kHz}$, respectively. The absolute error of $\mathrm{P}$-wave velocity is not more than $0.9 \%$, and that of S-wave velocity is not more than $0.6 \%$. FD-II type driven system of electrical resistivity was used for water drive.

\subsection{Experimental procedures}

The experiment was carried out according to the national oil and gas industry standard SY/T 6351-1998.

The surfaces of rock samples were first polished, next the samples were washed to remove oil and salt, and then dried and pretreated. Four kinds of experimental oils were selected including naphtha with density of $0.691 \mathrm{~g} / \mathrm{cm}^{3}$ and viscosity of $0.5-1.2 \mathrm{cP}$, light oil with density of $0.749 \mathrm{~g} / \mathrm{cm}^{3}$ and viscosity of 1.0-1.6 cP mixed by naphtha and mineral oil, mineral oil with density of $0.834 \mathrm{~g} / \mathrm{cm}^{3}$ and viscosity of 11.9 $12.7 \mathrm{cP}$, and hydraulic oil with density of $0.873 \mathrm{~g} / \mathrm{cm}^{3}$ and viscosity of 37.1-38.0 cP. The main experimental steps were as follows:

(1) P- and S-wave velocities of dry rock samples were measured by the ultrasonic pulse transmission method.

(2) The dry samples were saturated by brine with salinity of 26,000 ppm, and P- and S-wave velocities of water saturated samples were measured.
(3) The water saturated samples were dried and then saturated by one kind of experimental oil. The oil-water displacement experiment was made using FD-II system. At the designed saturation point, the sonic wave velocities of samples were measured.

(4) The samples were washed again to remove oil.

(5) Repeat steps (3) and (4) until all four kinds of oils were used for sonic wave velocity measuring with different oil/water saturations.

\section{Fluid replacement theory}

To compare the measured value with the theoretical value, the fluid replacement theory was used, which plays an important role on rock physics and is an important research tool both in the fluid identification and in quantitative reservoir analysis. The key of fluid replacement theory is Gassmann's equation (Mavko et al, 1998), described as:

$$
\begin{aligned}
& \frac{K}{K_{\mathrm{m}}-K}=\frac{K_{\mathrm{dry}}}{K_{\mathrm{m}}-K_{\mathrm{dry}}}+\frac{K_{\mathrm{f}}}{\Phi\left(K_{\mathrm{m}}-K_{\mathrm{f}}\right)} \\
& \mu=\mu_{\text {dry }}
\end{aligned}
$$

where, $K_{\mathrm{m}}, K_{\mathrm{f}}, K_{\mathrm{dry}}$ and $K$ are bulk moduli of rock matrix, pore fluid, dry rock and fluid-saturated rock, respectively; $\mu_{\mathrm{dry}}$ and $\mu$ are shear moduli of dry rock and fluid-saturated rock, respectively; $\Phi$ is porosity of rock. If the P- and S-wave velocities of dry rocks are known, P- and S-wave velocities of rocks with different fluid saturations can be calculated as follows:

(1) When the density $\left(\rho_{\mathrm{g}}\right)$ and P- and S-wave velocities $\left(V_{\mathrm{pg}}\right.$ and $V_{\text {sg }}$ ) of dry rock samples are known, $K_{\text {dry }}$ and $\mu_{\text {dry }}$ can be calculated by the following equations:

$$
K_{\mathrm{dry}}=\rho_{\mathrm{g}}\left(\left(V_{\mathrm{pg}}\right)^{2}-\frac{4}{3}\left(V_{\mathrm{sg}}\right)^{2}\right)
$$




$$
\mu_{\mathrm{dry}}=\rho_{\mathrm{g}}\left(V_{\mathrm{sg}}\right)^{2}
$$

(2) $K_{\mathrm{f}}^{(1)}$ of fluid 1 can be obtained by Wood's equation (Mavko et al, 1998); $K_{\mathrm{m}}$ can be calculated by the Voigt-ReussHill model. Set $K=K^{(1)}, K_{\mathrm{f}}=K_{\mathrm{f}}^{(1)}$, then $K^{(1)}$ of rock samples saturated by fluid 1 can be calculated by Eq. (1).

(3) $\operatorname{Keep} \mu^{(1)}$ equal to $\mu_{\text {dry }}$ :

$$
\mu^{(1)}=\mu_{\mathrm{dry}}
$$

(4) The P- and S-wave velocities $\left(V_{\mathrm{P}}^{(1)}, V_{\mathrm{S}}^{(1)}\right)$ of rock samples saturated by fluid 1 can be finally calculated with the measured $\rho^{(1)}$ :

$$
\begin{aligned}
& V_{\mathrm{p}}^{(1)}=\sqrt{\frac{K^{(1)}+\frac{4}{3} \mu^{(1)}}{\rho^{(1)}}} \\
& V_{\mathrm{s}}^{(1)}=\sqrt{\frac{\mu^{(1)}}{\rho^{(1)}}}
\end{aligned}
$$

where, $V_{\mathrm{P}}^{(1)}, V_{\mathrm{S}}^{(1)}, \rho^{(1)}, K^{(1)}$ and $\mu^{(1)}$ are P-wave velocity, $\mathrm{S}$-wave velocity, density, bulk modulus and shear modulus of rock samples saturated by fluid 1 , respectively, and $K_{\mathrm{f}}^{(1)}$ is bulk modulus of fluid 1 .

\section{Results and analysis}

\subsection{Relationship between sonic wave velocity and oil saturation of rock samples saturated by the same oil}

\subsubsection{Results}

In this study, two kinds of low-density oils and two kinds of high-density oils were used as the experimental oil. With the increase of oil saturation, the changing rules of $\mathrm{P}$ and S-wave velocities of rock samples saturated by the two low-density oils were similar, so only the results of rock samples saturated by the oil with density of $0.691 \mathrm{~g} / \mathrm{cm}^{3}$ were analyzed. In the same way, for rock samples saturated by two high-density oils, only the results of rock samples saturated by the oil with density of $0.834 \mathrm{~g} / \mathrm{cm}^{3}$ were analyzed.

The changing rule of $\mathrm{P}$ - and $\mathrm{S}$-wave velocities of rock samples saturated by the oil with density of $0.691 \mathrm{~g} / \mathrm{cm}^{3}$ was shown in Fig. 1(a). With the increase of oil saturation, P-wave velocity decreased, while S-wave velocity changed irregularly.

The changing rule of $\mathrm{P}$ - and S-wave velocities of rock samples saturated by the oil with density of $0.834 \mathrm{~g} / \mathrm{cm}^{3}$ was shown in Fig. 1(b). With the increase of oil saturation, $\mathrm{S}$-wave velocity also changed irregularly, but $\mathrm{P}$-wave velocity increased.

\subsubsection{Comparison of measured and theoretical values of sonic wave velocity}

Fluid replacement analysis of the four rock samples with different oil/water saturations was performed. The sonic wave velocities of dry rock samples were measured by experiment, and then $K_{\text {dry }}$ was acquired by Eqs. (3) and (4). Set $K_{\mathrm{w}}=2.279$ $\mathrm{GPa}, K_{\mathrm{f}}$ of the four kinds of oils with density of $0.691 \mathrm{~g} / \mathrm{cm}^{3}$,

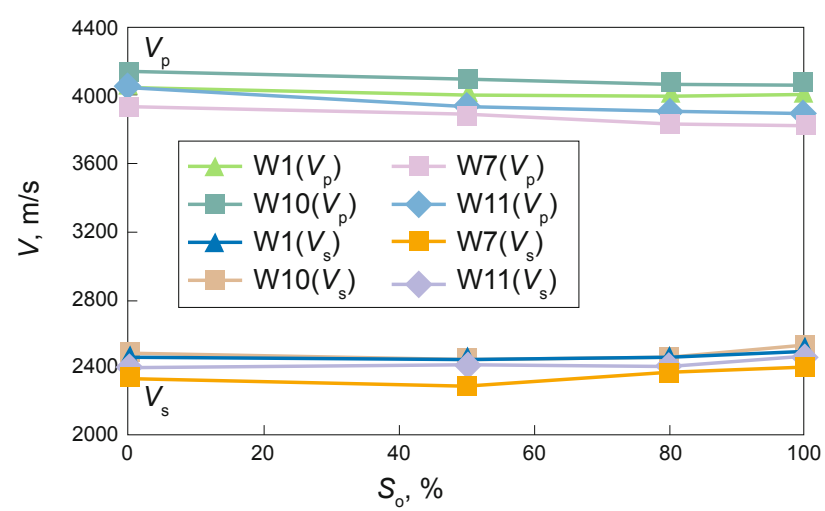

(a) Oil density is $0.691 \mathrm{~g} / \mathrm{cm}^{3}$

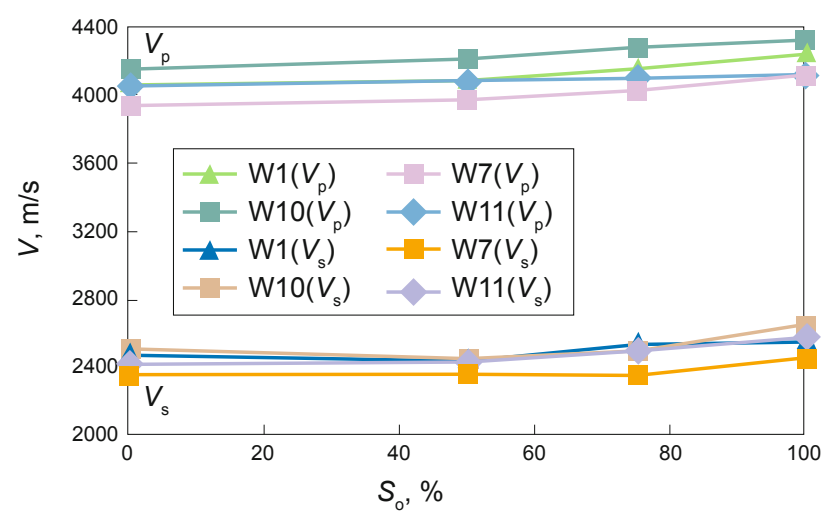

(b) Oil density is $0.834 \mathrm{~g} / \mathrm{cm}^{3}$

Fig. 1 Graphs showing the relationship between $V$ and $S_{0}$

$0.749 \mathrm{~g} / \mathrm{cm}^{3}, 0.834 \mathrm{~g} / \mathrm{cm}^{3}$ and $0.873 \mathrm{~g} / \mathrm{cm}^{3}$ is $0.993 \mathrm{GPa}$, $1.209 \mathrm{GPa}, 1.582 \mathrm{GPa}$ and $1.777 \mathrm{GPa}$, respectively. Thus, the sonic wave velocities of rock samples with different oil/water saturations can be calculated by the fluid replacement method mentioned above.

With the increase of oil saturation, both the measured and theoretical P-wave velocity of rock samples saturated by 0.691 $\mathrm{g} / \mathrm{cm}^{3}$ oil decreased, and the former was slightly larger than the latter (Fig. 2(a)). Generally speaking, the bulk modulus of rocks saturated by brine is greater than that saturated by low-density oil, but the shear modulus is almost unaffected by fluids. Density changes slightly with the increase of oil saturation. Thus, P-wave velocity decreases according to Eq. (6). The result that $\mathrm{S}$-wave velocity changes slightly with the change of oil saturation was consistent with the conclusion drawn by Shi et al (1995). The shear modulus was generally unchanged and the density of rock samples changed slightly with $S_{0}$, so the S-wave velocity changed slightly with $S_{\text {o }}$ according to Eq. (7). The measured P-wave velocity was larger than the theoretical value, because the theoretical value was derived from Gassmann's equation which is suitable for low-frequency sonic wave signals. The difference between the measured and the theoretical value was caused by acoustic dispersion (Shi and Yang, 2001). The fluid has a "nonrelaxation" effect on the high-frequency signals used in this experiment, and thus the rocks are in the undrained state and the stiffness of porous rock is large. 


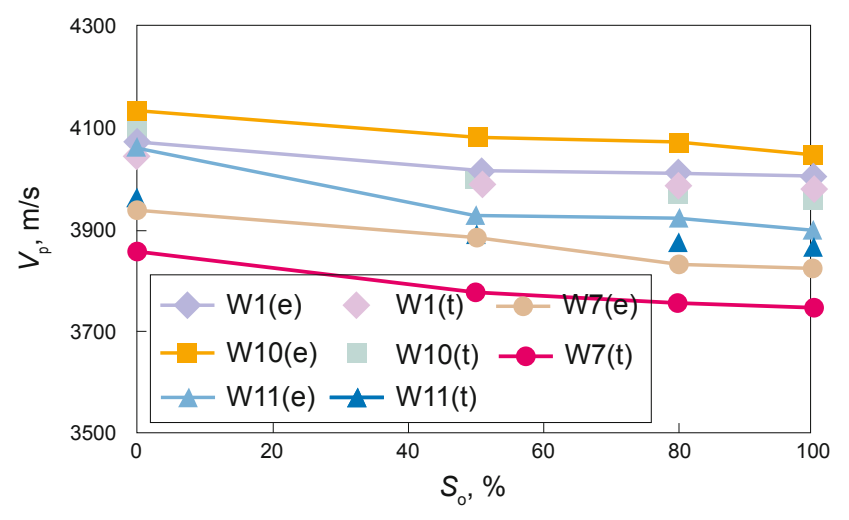

(a) Oil density is $0.691 \mathrm{~g} / \mathrm{cm}^{3}$

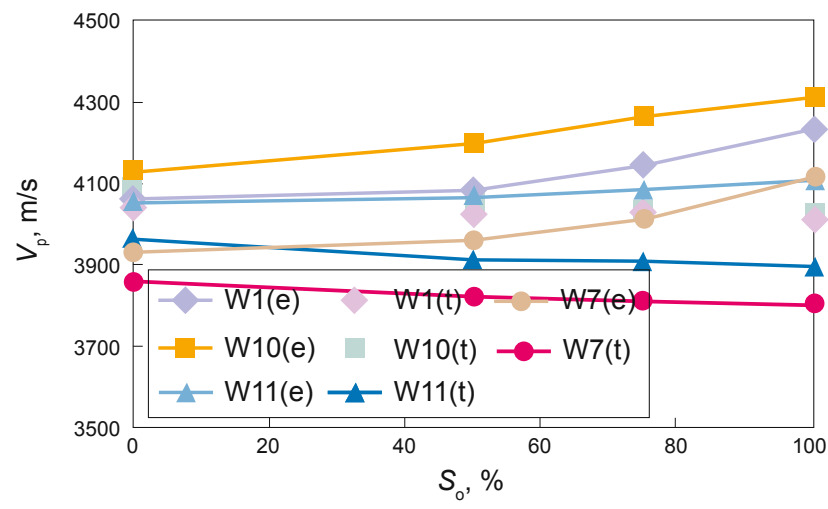

(b) Oil density is $0.834 \mathrm{~g} / \mathrm{cm}^{3}$

Fig. 2 Comparison of measured and theoretical P-wave velocity of rock samples with different oil saturations

(e refers to the measured value, and $t$ refers to the theoretical value)

With the increase of oil saturation, the measured P-wave velocity of rock samples saturated by $0.834 \mathrm{~g} / \mathrm{cm}^{3}$ oil increased, but the theoretical P-wave velocity decreased (Fig. 2(b)). The measured value of water-saturated samples was less than that of oil-saturated samples, which was inconsistent with the effective fluid theory. The effective fluid theory indicates that $\mathrm{P}$-wave velocity of water-saturated rock samples is greater than that of oil-saturated rock samples if the factors such as the cementation degree, the effect of pore fluid on rock skeleton, and the anisotropy of rocks are not taken into account. However, the actual situations are complex and do not match the above assumptions. A number of factors affect the results of this experiment, such as the pore structure, fluid distribution in pore space, interaction between fluid and rock skeleton, and the experimental frequency.

The result that the measured P-wave velocity is inconsistent with the theoretical value also occurred in the previous research. For example, the P-wave velocity of oilsaturated samples was greater than that of water-saturated samples in the experiment carried out by Shi and Yang (2001) and Huang et al (1998). The P-wave velocity of mineral oilsaturated sandstone samples was greater than that of watersaturated samples at a normal state (Xie et al, 1997). Shen and Shi (1994) found that the P-wave velocity of mineral oilsaturated samples was greater than that of brine-saturated samples.

There are three main reasons that lead to a contradiction between the experimental results and the theoretical results:

(1) The effect of water on the rock skeleton. There is hydrophilic clay in sandstone, and water is an infiltrating fluid. Water and clay interact with each other, resulting in the clay swelling and gaining of micro-pores. Thus, the crack density of rock increases, i.e., the porosity of rock increases from a microscopic view, and the P-wave velocity of watersaturated rock decreases as a result.

(2) The impact of fluid viscosity. The fluid viscosity decreases as the temperature increases, but the viscosities of mineral oil and hydraulic oil are still very large in the experimental temperature range. Therefore, the coupling effect between oil and rock skeleton is high and the rock stiffness increases. As the bulk modulus of oil-saturated rock increases, and the density of oil-saturated rock is less than that of water-saturated rock, the P-wave velocity of oilsaturated rock increases.

(3) The effect of acoustic dispersion. The dispersion effect of oil-saturated rocks is greater than that of water-saturated rocks.

For the S-wave, the experimental result showed that the changing rule is not regular. This is consistent with theory.

\subsection{Relationship between sonic wave velocity and oil density under the same oil saturation condition}

This experimental research of sonic wave velocities of rock samples saturated by oils of different densities was firstly reported in this paper. With the increase of oil density (namely $0.691 \rightarrow 0.749 \rightarrow 0.834 \rightarrow 0.873 \mathrm{~g} / \mathrm{cm}^{3}$ ), the P-wave velocity increased gradually and S-wave velocity changed little when the oil saturation was $100 \%$ (Fig. 3(a)). The changing rules of sonic wave velocities for the oil saturations of $75 \%$ and $50 \%$ were similar to the case of $100 \%$ saturation (Fig. 3(b) and 3 (c), note that the oil saturation was $80 \%$ for oil density of $0.691 \mathrm{~g} / \mathrm{cm}^{3}$ in Fig. 3(b)).

With the increase of oil density, the viscosity of highdensity oil increases. The coupling effect between pore fluid and rock skeleton is enhanced, so the bulk modulus increases, and the density of rock samples changes little. The sonic wave velocities of rock samples saturated by high-viscosity oil are greater than those of rock samples saturated by lowviscosity oil. Therefore, the P-wave velocity increases with the increase of oil density. The S-wave velocity changes little and irregularly, that is to say, S-wave is not sensitive to the fluid.

In the case of the same oil density, according to the relationship between sonic wave velocity and oil saturation of rock samples, the P-wave velocity of water-saturated rock samples was greater than that of oil-saturated rock samples when the oil density was $0.749 \mathrm{~g} / \mathrm{cm}^{3}$, but the P-wave velocity of water-saturated samples was less than that of oil-saturated samples when the oil density was $0.834 \mathrm{~g} / \mathrm{cm}^{3}$, and the change of S-wave velocity of all the rock samples was not regular.

In the case of the same oil saturation, according to the relationship between sonic wave velocity of rock samples and oil density, the P-wave velocity of rock samples increases 


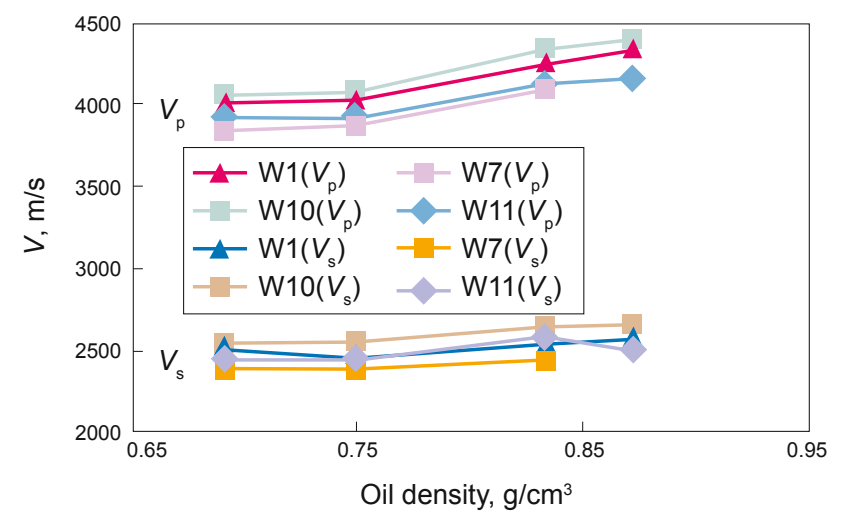

(a) Oil saturation is $100 \%$

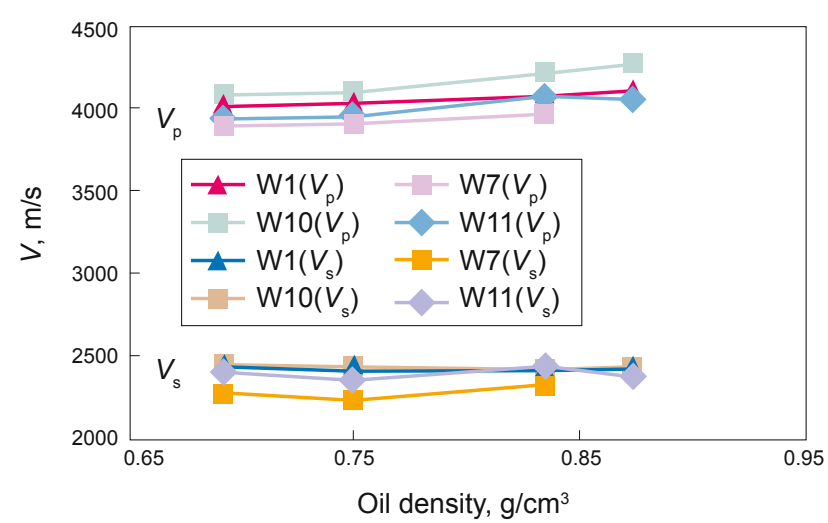

(b) Oil saturation is $75 \%$ or $80 \%$

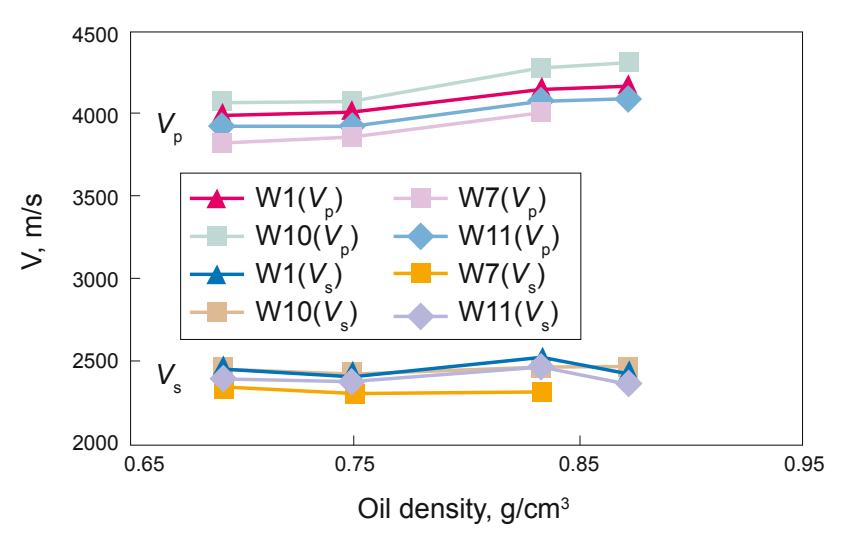

(c) Oil saturation is $50 \%$

Fig. 3 Sonic wave velocity of rock samples saturated by different oils (Note: when the sample W7 was saturated by $0.873 \mathrm{~g} / \mathrm{cm}^{3}$ oil, the data were doubtful due to large cracks)

with the increase of oil density, but the S-wave velocity changes little. Therefore, when the oil density changed from $0.749 \mathrm{~g} / \mathrm{cm}^{3}$ to $0.834 \mathrm{~g} / \mathrm{cm}^{3}$, there was a critical value $\rho_{\mathrm{c}}$. When the oil density was equal to $\rho_{\mathrm{c}}$, the P-wave velocity of water-saturated rock samples was equal to that of oilsaturated rock samples, and the properties of water-saturated rock samples were similar with those of oil-saturated rock samples. If the density of crude oil in a reservoir is equal to $\rho_{\mathrm{c}}$, the properties of oil-saturated rocks would be similar with those of water-saturated rocks, so it is difficult to distinguish hydrocarbon from water by conventional parameters. If the density of crude oil is less than or greater than $\rho_{\mathrm{c}}$, there will be differences among these parameters, such as sonic wave velocities and rock density. The greater the differences, the better the effects on the hydrocarbon detection.

\section{Conclusions}

1) When oil saturation increases, the measured P-wave velocity of rocks saturated by low-density oils decreases and is close to the theoretical value, but increases when rocks are saturated by high-density oils and is inconsistent with the theoretical value. This contradiction might result from the physical and chemical interactions between pore fluid and rock skeleton, the effect of viscosity of high-density oil, acoustic dispersion, pore geometry, oil/water saturation and measuring frequency. The change of S-wave velocity is not regular.

2) When oil saturation is constant, the P-wave velocity of rocks increases gradually with the increase of oil density (namely $0.691 \rightarrow 0.749 \rightarrow 0.834 \rightarrow 0.873 \mathrm{~g} / \mathrm{cm}^{3}$ ), but the S-wave velocity changes little. This result is valuable for hydrocarbon detection.

3) The changing rule of $\mathrm{P}$ - and S-wave velocities of rocks is valuable for pre-stack seismic attribute analysis and seismic inversion, and can be used in reservoir prediction in the study area.

\section{Acknowledgements}

The research was supported by the Science and Technology Research Key Project of Ministry of Education (Grant No.109035), the National Natural Science Foundation Key Project (Grant No.40830423) and the Key Project of Students Extra-curricular Science and Technology Research Program of Schlumberger (Grant No.SLBX0908). We would like to express our appreciation to director Yu Jun, engineer Zhou Jianqiao, Yang Fang from the Rock Physics Laboratory of Daqing Well Logging Company for their assistance in the experiment.

\section{References}

Deng J X, Wang S X and Yu J. Experimental results in partially saturated sandstone under condition of different pressure and their theoretical interpretation. Oil Geophysical Prospecting. 2005. 40(5): 530-534 (in Chinese)

Domenico S N. Effect of water saturation on seismic reflectivity of sand reservoirs encased in shale. Geophysics. 1974. 39(6): 759-769

Goertz D and Knight R. Elastic wave velocities during evaporative drying. Geophysics. 1998. 63(1): 171-183

Huang K, Xu Q Z, Yang X H, et al. Oil, gas and water distribution detection forward modeling with elastic parameters of core samples under simulated condition. Chinese Journal of Geophysics. 1998. 41(Supplement): 414-421 (in Chinese)

Hu X H, Li C W, Li X, et al. Experiments on acoustic characters of low porous and permeable sandstones. Well Logging Technology. 2004. 28(4): 273-276 (in Chinese)

Jiang L X and Shi X J. Relation between wave velocity in sandstone and fluid content in porous medium under high frequency condition. Oil Geophysical Prospecting. 1998. 33(3): 355-362 (in Chinese) 
Jiang W Z, Zhang C G, Huang W X, et al. Acoustic characteristics of core under high temperature and high pressure. Journal of Jianghan Petroleum Institute. 2004. 26(1): 51-53 (in Chinese)

Knight R and Nolen-Hoeksema R. A laboratory study of the dependence of elastic wave velocities on pore scale fluid distribution. Geophysical Research Letters. 1990. 17(10): 1529-1532

Liu Z P, Wu X W and Chu Z H. Laboratory study of acoustic parameters of rock. Chinese Journal of Geophysics. 1994. 37(5): 659-666 (in Chinese)

Mavko G, Mukerji T and Dvorkin J. The Rock Physics Handbook. New York: Cambridge University Press. 1998. 71-90

Shen L D and Shi G. Effect of lithologic character, petroleum and effective overburden pressure on compressional wave and shear wave velocity. Chinese Journal of Geophysics. 1994. 37(3): 391-398 (in Chinese)

Shi G and Shen L D. Experimental study of how water saturation in limestone influences the velocities of $\mathrm{P}$ wave and $\mathrm{S}$ wave. Oil Geophysical Prospecting. 1990. 25(4): 469-479 (in Chinese)

Shi G and Yang D Q. Determination of the elastic wave velocities in porous rocks with the change of overburden pressure and its universal significance. Science in China (Series D). 2001. 31(11): 896-901 (in Chinese)

Shi G, Shen W L and Yang D Q. The relationship of wave velocities with saturation and fluid distribution in pore space. Chinese Journal of Geophysics. 2003. 46(1): 138-142 (in Chinese)

Shi X J, Xu G M, Jin P, et al. The laboratory study of influence of water saturation on rock's velocity and attenuation. Chinese Journal of Geophysics. 1995. 38(Supplement 1): 281-287 (in Chinese)

Xie J Z, Chu Z H and Liu Z P. A study on acoustic characteristics of oil-saturated sand in the process of water flooding. Well Logging Technology. 1997. 21(6): 401-404 (in Chinese)

Xu Q Z, Wu J H and Zhang L F. Rock wave velocity and resistivity in fluid flooding process under stratal condition. Geophysical Prospecting for Petroleum. 2000. 39(1): 124-126 (in Chinese)

Zhang Y H and Li C W. Experimental study of the relation between specific property of P-wave and water saturation in rock. Well Logging Technology. 1995. 19(1): 6-10 (in Chinese)

Zhang Y H, Hu X H and Li C W. Experiments on identification of gas in sandstone formation with acoustic parameters. Well Logging Technology. 2000. 24(3): 194-197 (in Chinese)

Zhao F Z, Cai M L and Sai F Y. Experimental study on acoustic wave and resistivity of rock under high temperature and pressure. Well Logging Technology. 1998. 22(Supplement): 3-5 (in Chinese)

(Edited by Hao Jie) 